\title{
Ren Zhao: no standard answer to be a doctor
}

Submitted Dec 17, 2019. Accepted for publication Jan 16, 2020.

doi: 10.21037/atm.2020.01.121

View this article at: http://dx.doi.org/10.21037/atm.2020.01.121

"What is most interesting for a doctor in the end? Nobody can be your teacher, and books cannot help you; you must discuss with team members and find an answer. There is no standard answer, especially when you are dealing with some complicated problems; you have to find a new way to solve the problems, and that is the most interesting thing to be a doctor."

-Ren Zhao

Executive Vice President of Ruijin Hospital North

\section{From result to process}

Measured by the result, Prof. Ren Zhao is successful.

In 1995, he stood out from a dozen students to be the only one enrolled in a successive postgraduate and doctoral program at Ruijin Hospital.

With some 30 years of experience in minimally invasive surgery for colorectal cancer, he completed the first case of radical treatment of rectal carcinoma with the combination of laparoscopic surgery and intraoperative radiotherapy in 2012; in 2013, he successfully performed the singleport laparoscopy-assisted radical resection of colorectal carcinoma, which was a globally leading technique. So far, he has performed da Vinci robot-assisted radical resection of colorectal carcinoma in over 250 patients.

At 47 , he became the executive vice president of the Ruijin Hospital North (RHN) and executive director of the Department of General Surgery. The new roles represent a path of integration for him as a doctor and a manager.

However, Ren Zhao likes to do things that appear "useless". For instance, when he does not work, he would go to the library and read books about humanity and history, hoping to find an answer to a question from another perspective.

He has also received some "useless" training. From 1997 to 2010, he went to Germany, France, the United Kingdom, and the United States for further studies, and most of the training sessions were not well known. These new techniques included regional chemotherapy techniques for gastrointestinal tumors, multidisciplinary treatment of liver metastases of colorectal cancer, transperineal sphincter- preserving surgery for ultra-low rectal carcinoma, and radiotherapy techniques for gastrointestinal tumors.

"What I have learned was somewhat less popular knowledge." Recalling his training experience, Ren Zhao said, "taking intraoperative radiotherapy as an instance, and it remains an option for a small number of patients; it is only feasible for patients with the small and localized recurrence of tumors near some vital organs, and the tumors cannot be completely removed through surgery".

Whether the technologies are popular or unpopular, Ren Zhao has never set any limits for himself. From his perspective, medical science has been progressing based on repeated attempts.

Last year Ren Zhao traveled to Italy for academic exchange with an Italian Ministry of Health-affiliated research institute, home of two Nobel Prize winners. When he asked about its research experience, the institute head replied that they would do research as long as it is scientifically justified, with good workflow, and in line with the developmental trends of medical science. "It is not our major concern about whether the research will produce tangible results." Said the head. The view has long been echoed and shared by Ren Zhao.

"For me, nothing is off-limit, and we can watch and wait. Training experiences in four countries have been extremely helpful for me, and I am also exploring new means and approaches to treat colorectal diseases." (Figure 1).

\section{From five ports to one port}

Since radical laparoscopic surgery for colorectal cancer was performed in 2009, Ren Zhao has been pioneering innovations from five ports to three ports and then a single port. For him, "reducing the number of ports is not the goal but offers a new option. The goal is to bring better treatment outcomes to the patients".

The first attempt for Ren Zhao to perform a reducedport surgery was to improve a leaky gut.

The five-port laparoscopic surgery for colorectal cancer usually requires three people to complete-the operator, 


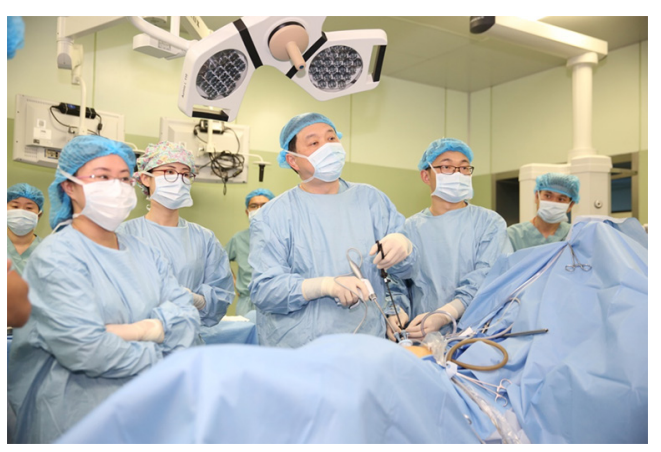

Figure 1 Dr. Ren Zhao is performing a surgery.

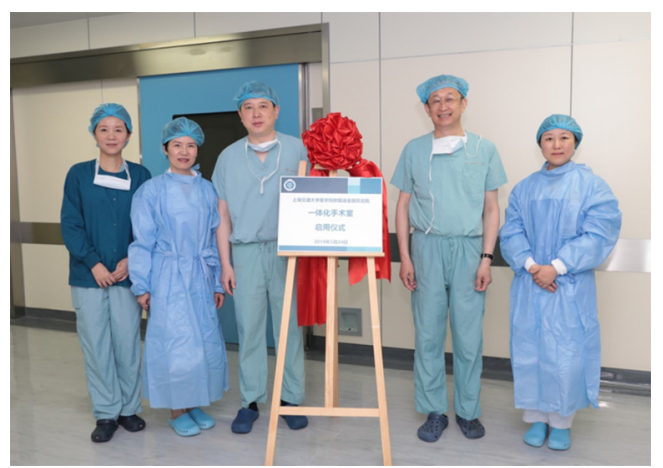

Figure 2 In May 2019, the first full HD integrated operating room of the Ruijin Hospital North was officially launched (Third from the left: Ren Zhao).

the camera holder, and the assistant. When the five-port technique was introduced, Ren Zhao found the incidence of intestinal leakage was slightly higher than the conventional open surgery, which was not due to the poor suturing at the anastomosis but because the intestinal wall near the anastomosis had small tears or the mesenteric blood vessels were damaged, which resulted in intestinal ischemia and thus led to intestinal leakage. "This was often caused by the poor cooperation between the assistant and the operator, and excessive force was applied during traction.”

In an occasional surgical operation, Ren Zhao completed the dissection of the rectum, dissection and transection of the roots of the blood vessels, and separation of the anterior iliac fascia and Denonvillier fascia with the presence of only one camera holder. "This inspired us. Since two people could also complete the surgery, why should we use five ports?”

Immediately, Ren Zhao launched clinical studies on reduced port surgery in the open cohorts. They started with three ports but were not restricted to three ports; when the operation met difficulties, new ports would be added one by one or even converted to open surgery. Their studies revealed that patients undergoing three-port laparoscopic surgery not only recovered quickly after surgery, but also reduced the time, consumables, and labor costs of the operation.

In 2013, Ren Zhao successfully launched the world's leading single-port laparoscopic radical mastectomy for colorectal cancer based on reduced-port surgery. "In the past, five ports plus a $5-\mathrm{cm}$ incision in the abdominal wall where needed, and today only one $3-\mathrm{cm}$ incision is needed, which offers better cosmetic results and allows faster recovery. Patients often believe that they have just undertaken a 'small operation', which is quite helpful for postoperative mental recovery." Said Ren Zhao. At present, single-port laparoscopic radical resection of colorectal cancer accounts for more than $20 \%$ of surgeries at RHN.

Ren Zhao also has carried out clinical research on radical robotic resection of colorectal cancer using da Vinci surgical system, sphincter-sparing resection, and adjuvant treatment for ultra-low rectal cancer, and preoperative adjuvant chemotherapy, radiotherapy and intraperitoneal chemotherapy for middle and low rectal cancer.

"The changes in surgical procedures depend on the changes in doctors' understanding of technology. Whenever a new surgical technology is mentioned, people always want to know what the indications are. For reduced-port surgery, there are no absolute indications, and everything can be flexibly dealt with according to changes in specific conditions." So, in Ren Zhao's opinion, surgeons must have an open mind and be ready to accept new things (Figure 2).

In 2017, Ren Zhao was awarded the Fellow of the American College of Surgeons (FACS) at the $103^{\text {rd }}$ American College of Surgeons Clinical Congress in recognition of his achievements and contributions to surgery.

Currently, Ren Zhao is exploring how to integrate artificial intelligence with surgical robots. "The coming fifth-generation da Vinci Surgical System will be based on a single port, and we hope to graft a digital surgical mode inside it, allowing the more intelligent surgical robot imitates a doctor to perform surgery."

\section{From treatment to prevention}

The prevalence and mortality of colorectal cancer have shown a rising trend in recent years. Despite the improved 


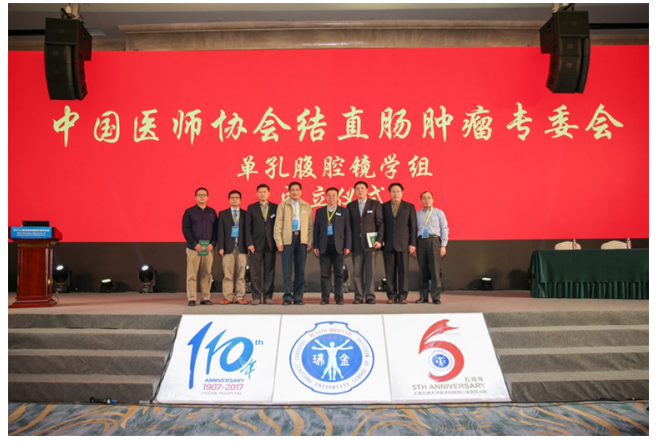

Figure 3 The Professional Committee on Single-port Laparoscopy of the Colorectal Cancer Professional Committee of the Chinese Medical Doctors Association was set up in December 2017, and Dr. Ren Zhao (third from the right) serves as the first chairman.

clinical diagnosis and surgical treatment capabilities, the 5 -year overall survival of colorectal cancer is still only about $50 \%$ in China.

Most patients are already in the advanced stages at the time of diagnosis, and the treatment response is still low. At present, only $10 \%$ of colorectal cancer cases are diagnosed in their early stages. How to increase the survival rate and improve the quality of life has long been the top priority in RHN.

If more patients with advanced colorectal cancer can be found early, it will not only dramatically lower the mortality rate but also effectively reduce the medical expenses of the whole society. To this end, Ren Zhao and his team have established a standardized early colorectal cancer diagnosis system in RHN: simple, practical, and operable screening are performed among outpatients, and the early diagnosis rate of colorectal cancer has been increased through "opportunistic screening".

"We perform a fecal occult blood test and a Colorectal Cancer Risk Assessment Tool-based scoring on the outpatients. If the patients are at elevated risk, colonoscopy and pathology will be performed to confirm the diagnosis, to ensure correct preoperative staging and treatment." After several years of practice, up to $90 \%$ of outpatients have received screening for colorectal diseases, and the accuracy of the preoperative staging of colorectal cancer for inpatients reached $100 \%$.

"Today, thanks to the implementation of early screening, the prevalence and mortality rates of colorectal cancer are decreasing in the United States." Introduced Ren Zhao. In Shanghai, China, the Community Residents Colorectal Cancer Screening Program, a major municipal public health project, has entered the second round. "Hopefully, this program will become a routine practice in Shanghai. It will identify more patients with polyps and early bowel cancer and thus benefit them when the diseases are still in their early stages."

In 2015, the "Research on Optimized Diagnosis and Treatment of Colorectal Cancer", with Ren Zhao being the principal investigator, won the Shanghai Medical Science and Technology Award (third prize). Based on the existing methods, this study explored several key points in colorectal cancer treatment, including prompt treatment, graded treatment, improvements in minimally invasive technology, and prognostic evaluation. The optimize the treatment, the study explored the values of opportunistic screening and extended indications of traditional minimally invasive surgery and investigated the mechanism of metastasis and recurrence, with an attempt to achieve better outcomes and benefit more patients (Figure 3).

\section{From a doctor to a manager}

RHN opened for service in December 2012 as part of efforts to push for a balanced distribution of high-quality medical resources in Shanghai. The new hospital marked a significant turning point for Ren Zhao-he became executive vice president of RHN, in a transition from a surgeon to a manager.

The memory of the opening ceremony is still fresh for him. "It was a very fantastic day. It was snowing before the opening ceremony. When the ceremony began at 10 a.m., the snow stopped falling, the wind stopped blowing, and the sun came out of the sky." Everything has shown a good start.

Ren Zhao had taken managerial roles as early as 2008.

The deadly earthquake striking Wenchuan on May 12 had caused serious damages to the nearby Dujiangyan city. When Ren Zhao learned that Shanghai Health Bureau was assembling a medical team to be sent to Dujiangyan, he signed up in the first place to join the team.

In this trip, he not only served as the chief of the medical team from Ruijin Hospital to support the reconstruction of Dujiangyan after the disaster but also was the only clinician in the "Expert Working Group for the Recovery of Medical Orders and Medical Capabilities after the Earthquake in Dujiangyan", during which he developed a series of postdisaster health reconstruction plans for the local areas.

"This has been a rare experience in my life." For the first time, Ren Zhao was more than a doctor. He took part 
in hospital management, made important decisions, and oversaw hospital constructions, and he was dealing with patients, government officials, and people from all walks of life. The experience allowed him to have a more profound understanding of the hospital's functions and ordinary citizens' real needs. "In the past, I thought a good doctor's job is to heal the wounded and rescue the dying, and he/she should have extraordinary medical skills. However, most of us have ignored the fact that a hospital's fundamental role is to provide good medical services."

As the executive vice president of RHN, Ren Zhao aims to provide patients with the same quality of medical services as the headquarter Ruijin Hospital.

To that end, Ren Zhao invited Ruijin Hospital's department directors to head the same departments at RHN and created new roles of executive vice directors to replace the directors from the headquarter hospital gradually. Besides, young doctors will receive training at Ruijin Hospital under the same standards and the same culture, to cultivate their benevolence and the pursuit for excellence.

Based on medical demands in Jiading District, where RHN is found, Ren Zhao has sent experts and professors to supply medical instructions on the front-line. In the meantime, RHN has made proper adjustments in disease structure, department settings, and hospital management to ensure its sustainable development.

Ren Zhao admitted that RHN is still trailing Ruijin Hospital in terms of clinical disciplines and medical resources, but the new facility offers fresh opportunities for many professors, experts, young doctors, and even managerial officers. For instance, RHN is currently focused on the featured therapies for tumors.

"RHN is in a critical turning point now, and based on providing services to patients in the Jiading District, and we should strive to expand our reach to the Yangtze River Delta region. With the inauguration of Ruijin Hospital's Proton Therapy Center and RHN's phase II expansion, the new center could be developed into a tumor-focused general hospital."

\section{Epilogue}

Ren Zhao sees himself as a lucky person. His good luck is proved in two aspects. On the one hand, he is lucky to have received help and instructions from several elder experts including Ruijin Hospital President Hong Li and Prof. Baoming Yu, and their words and deeds have deepened his understanding of the hospital's spirit of pursuing benevolence and excellence; on the other hand, he is lucky to be appointed as executive vice president of RHN and take charge of day-to-day management.

"This is a hard-earned experience for me because I can do something that I had no chance to do in the past. In the past, I only thought about doing research work and performing surgeries, and now I have to manage the hospital well and make it a famous and extensively influential general hospital; also, I must help our doctors advance their careers."

Recently, Ren Zhao has developed an interest in the development history of hospitals. In an ever-changing society, he is not sure about the future of surgery or RHN. However, he is sure that he will continue to move ahead and think about what to do in the future.

"There is no standard answer in medical science, and there is no standard answer in our life. We will get the answer after a try."

\section{Dialogue Prof. Ren Zhao}

\section{AME: What is organ function preservation?}

Ren Zhao: The organ function preservation is to support the normal function of a specific organ. It has long been a belief that surgery will unavoidably injure the organ(s). With the availability of more sophisticated or precise surgeries, the organ function (e.g., urinary function, reproductive function, and anal function) can be preserved to the maximum extent.

\section{AME: What are the key points of anal sphincter preservation surgery?}

Ren Zhao: First, we need to completely resect the bowel cancer, which is the premise of anal sphincter preservation. Second, the function of the anus must be good after the sphincter preservation. Otherwise, the procedure would be nonsense if the patient must pass stool 10 or even 20 times a day. This also explains the value of ultra-low sphincter-saving procedures.

\section{AME: How to properly understand intestinal stomas?}

Ren Zhao: Artificial anus, also known as the artificial anus or intestinal stoma/intestinal fistula, is to change the route via which the stool is passed. For patients, the artificial anus is inconvenient in the short term but will eventually achieve a higher quality of life than a barely-preserved anus, as confirmed by a large amount of evidence. Therefore, we should consider the anal sphincter preservation surgery 
objectively, scientifically, and rationally.

\section{AME: What is the opportunistic screening for outpatients?}

Ren Zhao: Opportunistic screening refers to screen patients at elevated risk of tumors based on available clues. For these patients, the fecal occult blood test is performed firstly; if the result is positive, the patients will be inquired for their demographic data, medical histories, and family histories. Outpatients meeting specific conditions will receive a secondary diagnosis process, that is, colonoscopy.

Screening for gene expressions is also available in our center. Some methylated genes of tumors may enter the stool, and rinsing, extracting, and screening of these genes may achieve an accuracy rate of $80 \%$.

Also, patients with a history of inflammatory gastrointestinal diseases or familial polyps or other obvious gastrointestinal symptoms (such as changes in stool shape) require further examinations. Patients with a family history of bowel cancer typically have ten times the risk of developing bowel cancer than normal people.

\section{Profile}

Prof. Ren Zhao, MD, FACS, doctoral supervisor, is the Executive Vice President of RHN, an affiliated hospital of Shanghai Jiaotong University School of Medicine, and also the Executive Director of the Department of General Surgery, RHN.

$\mathrm{He}$ is a member of the Anorectal Group of the Chinese Medical Doctors Association, a member of the Standing Committee of the Colorectal Special Committee of Chinese College of Surgeons, chairman of the Single-port Laparoscopy Group of the Colorectal Cancer Professional Committee of the Chinese Medical Doctors Association, member of the Director Board of Shanghai Hospital Association, vice chairman of the Professional Committee on Colorectal Cancer of Shanghai Anti-Cancer Association, member of the General Surgery Committee of Shanghai Medical Association and deputy Leader of the Anorectal Group, vice chairman of the ERAS Specialist Committee of the Professional Committee on Colorectal Diseases of Chinese Medical Association, member of the Robotics Specialist Committee of the Specialty Committee on Colorectal Diseases of the Colorectal Surgery Branch of Chinese Medical Doctors Association.

$\mathrm{He}$ also serves as a member of the editorial boards of many academic journals, including the International Journal of Surgery. He has won one National Science and
Technology Progress Award (third grade), one Shanghai Medical Science and Technology Award (third grade), and one Jiading District Scientific and Technological Progress Award (first grade) (including the honorable title of "Leading Talent in Jiading District". He has taken part in one National 973 Program-supported research and was the principal investigator of two studies supported by the National Natural Science Foundation of China. He has also earned grants from the Shanghai Science and Technology Commission, Shanghai Municipal Health Commission, and the National Drug Administration. He has published 100 scientific articles in SCI-indexed journals and other core journals. He authors several books, including one of the nationally planned textbooks-Anorectal Diseases. He has been invited to give lectures at many important conferences.

\section{Acknowledgments}

Funding: None.

\section{Footnote}

Provenance and Peer Review: This article was commissioned by the Editorial Office, Annals of Translational Medicine. The article did not undergo external peer review.

Conflicts of Interest: The authors have no conflicts of interest to declare.

Open Access Statement: This is an Open Access article distributed in accordance with the Creative Commons Attribution-NonCommercial-NoDerivs 4.0 International License (CC BY-NC-ND 4.0), which permits the noncommercial replication and distribution of the article with the strict proviso that no changes or edits are made and the original work is properly cited (including links to both the formal publication through the relevant DOI and the license). See: https://creativecommons.org/licenses/by-nc-nd/4.0/.

(Science Editors: Siying Yan, Chen Gao, ATM, editor@atmjournal.org)

(Language Editor: Chapnick Jeremy Dean, AME Publishing Company)

Cite this article as: Yan S, Gao C. Ren Zhao: no standard answer to be a doctor. Ann Transl Med 2020;8(5):257. doi: 10.21037/atm.2020.01.121 